\title{
Clinician Delivery of Virtual Pivotal Response Treatment with Children with Autism during the COVID-19 Pandemic
}

\author{
Devon M. White ${ }^{1}$, Claire Aufderheide-Palk ${ }^{1}$ and Grace W. Gengoux ${ }^{2, *(D)}$ \\ 1 Division of Child \& Adolescent Psychiatry, Stanford Children's Health, Stanford, CA 94305-5719, USA; \\ dewhite@stanfordchildrens.org (D.M.W.); caufderheide@stanfordchildrens.org (C.A.-P.) \\ 2 Department of Psychiatry and Behavioral Sciences, Stanford University School of Medicine, \\ Stanford, CA 94305-5719, USA \\ * Correspondence: ggengoux@stanford.edu
}

\section{check for} updates

Citation: White, Devon M., Claire Aufderheide-Palk, and Grace W. Gengoux. 2021. Clinician Delivery of Virtual Pivotal Response Treatment with Children with Autism during the COVID-19 Pandemic. Social Sciences 10: 414. https://doi.org/ 10.3390/socsci10110414

Academic Editors: Eduardo Bunge, Blanca Pineda, Taylor N. Stephens, Naira Topooco and Nigel Parton

Received: 1 September 2021

Accepted: 26 October 2021

Published: 29 October 2021

Publisher's Note: MDPI stays neutral with regard to jurisdictional claims in published maps and institutional affiliations.

Copyright: (c) 2021 by the authors. Licensee MDPI, Basel, Switzerland. This article is an open access article distributed under the terms and conditions of the Creative Commons Attribution (CC BY) license (https:// creativecommons.org/licenses/by/ $4.0 /)$.

\begin{abstract}
This concept paper describes how the evidence-based Pivotal Response Treatment (PRT) model of autism treatment was adapted for delivery via telehealth during the COVID-19 pandemic. Multiple features of existing video conference technology were utilized for implementation of PRT motivational strategies with young children with autism spectrum disorder (ASD). PRT is an empirically supported treatment for ASD which can be taught to parents and delivered directly by trained therapists. During the COVID-19 pandemic, when the majority of clinical care for children with ASD had to be transitioned to telehealth delivery, models for parent training had been previously established. However, no model for direct clinician delivery of virtual PRT existed. This manuscript outlines practical details of the model developed by our team and provided to a total of 17 families between April 2020 and May 2021. Key lessons from technological adaptations of the PRT motivational strategies are described in order to inform future empirical investigation of this approach. The virtual PRT delivery model can serve as a guide for engaging children in meaningful social interaction and communication practice via video conference software, with implications for expanding access to autism treatment as well as for motivating a wide range of children in distance learning activities.
\end{abstract}

Keywords: autism; pivotal response; telehealth; COVID-19; virtual

\section{Introduction}

Early and intensive behavioral intervention is considered medically necessary for many children with Autism Spectrum Disorder (ASD) due to the consistent finding that early intervention and acquisition of functional language at a young age improve outcomes (Mouga et al. 2020; Posar and Visconti 2019) and that interventions implemented daily within natural routines have a particularly positive effect (Koegel et al. 2014). Pivotal Response Treatment (PRT) is one such intervention frequently sought-after by families of children with ASD due to its emphasis on child motivation and evidence for effectiveness in improving functional and social communication skills (Koegel and Koegel 2019). This developmental and child-directed approach to implementing established principles of ABA in naturalistic settings has been identified as an effective intervention for pre-school and elementary-aged children with ASD (National Research Council 2001; National Autism Center 2015; Steinbrenner et al. 2020). In PRT, motivation is encouraged through antecedent procedures (following the child's lead, providing choices, interspersing easy tasks) and consequence strategies focused on establishing natural relationships between the child's behavior and the reinforcer. The combination of parent training to implement evidencebased early intervention supplemented by clinician-delivered implementation of treatment is consistent with established best practices in ASD care and an effective way of delivering PRT (Gengoux et al. 2019). 
Prior to the COVID-19 pandemic, an emerging body of literature supported the application of video conferencing technology as a promising method for eliminating physical barriers to accessing in-person services, facilitating timely access to treatment within critical periods of development and cost-effective delivery for families with children at risk or diagnosed with developmental disorders (Simacek et al. 2021; Lindgren et al. 2016). Additionally, there was emerging evidence that parent training in naturalistic developmental behavioral interventions such as PRT, the Early Start Denver Model (ESDM), and the Joint Attention, Symbolic Play, Engagement, and Regulation (JASPER) approach could be effectively provided via online and video-based technology with positive results in terms of both parent and child learning (McGarry et al. 2020; Nefdt et al. 2010; Vismara et al. 2016; Pacia et al. 2021). However, little evidence of direct delivery of social communication intervention between clinicians and children with ASD via telehealth existed, meaning that many young children lost their direct care when stay-at-home orders limited in-person treatment at the onset of the COVID-19 pandemic. Some preliminary findings are now emerging on this topic, showing positive effects for interventions when delivered by a therapist through video conferencing technology (Cihon et al. 2021). For PRT specifically, a model for therapist delivery via telehealth still does not exist.

This paper describes one early intervention clinic's response to pandemic-related restrictions on in-person PRT delivery, which resulted in the development of a telehealth model for facilitating PRT via a virtual platform. In this manuscript, we detail the innovative ways that PRT therapists in our academic medical center capitalized on the necessity for technology-based PRT delivery to improve aspects of a treatment which is traditionally delivered only in face-to-face interactions. Additional resources and training materials related to PRT implementation for therapists and parents are available on the program website (https:/ / med.stanford.edu/autismcenter/prt.html, accessed on 1 September 2021). Through adapting clinician-delivered PRT to a telehealth model, therapists were able to continue to provide needed therapy during COVID-19 stay-at-home orders and clinic closures. Further, therapists utilized digital resources and video conference technology in innovative ways to deliver treatment with fidelity and efficacy via the virtual platform, and to help patients make progress toward their critical social communication goals. Lessons learned from implementation over more than 1460 sessions provide critical insights for both in-person and continued telehealth treatment of ASD, as well as implications for the likely inevitable integration of technology into ASD early intervention practice in the future.

\section{Background}

\subsection{Overview of Pivotal Response Treatment}

PRT is an established treatment for ASD with documented beneficial effects for young children in communication, social interaction, play, learning, and adaptive skills (Koegel and Koegel 2019). For instance, recent reviews of evidence-based treatment for young children with autism support the use of PRT to teach communication skills, joint attention, play, social, cognitive, and school readiness skills (National Autism Center 2015; Steinbrenner et al. 2020). PRT has also been associated with improvement, generalization, and initiations of social skills that were targeted in treatment (Verschuur et al. 2014). PRT is most often applied to children with ASD from toddlers to school-age and can be applied with children who have a wide range of verbal and cognitive skills, including children who are nonverbal or have below average intellectual or developmental levels as well as children who are verbally fluent or have average or above average intellectual ability (Hardan et al. 2015; Mohammadzaheri et al. 2014). This treatment is classified as a naturalistic developmental behavioral intervention (NDBI) because it incorporates both evidence-based applied behavior analysis (ABA) principles and play-based, child-directed developmental teaching strategies applied across natural environments (Schreibman et al. 2015). PRT focuses on foundational pivotal areas of development such as child motivation, yielding global improvement in other areas of communication (Bruinsma et al. 2019). While parent training is a critical component of PRT, clinician-delivered intervention is often used 
to supplement parent delivery, with beneficial effects for parents and children (Gengoux et al. 2019; Schreibman and Stahmer 2014).

\subsection{Core PRT Intervention Strategies}

The foundational motivational strategies of PRT include three core antecedent strategies-(1) child attention and clear opportunities, (2) interspersing maintenance and acquisition tasks, and (3) child choice and shared control—along with three core consequence strategies-(4) contingent reinforcement, (5) natural reinforcement, and (6) reinforcing attempts (Koegel and Koegel 2012). In a clinician-delivered PRT session, the therapist typically starts by observing the child playing in order to determine a potential activity of interest (child choice). The therapist then finds a playful way to briefly interrupt the play, often by gaining physical control of a preferred toy or introducing a novel but related item (shared control). If the child remains motivated despite the subtle disruption, the therapist ensures the child is attending to the toy or therapist (child attention) before prompting (clear opportunity) a verbal response. The child is expected to respond to the prompt and then receives the item (natural reinforcement) immediately following their verbal response (contingent reinforcement). Opportunities to practice functional communication are deliberately sequenced to enhance motivation through frequent successful practice of learned skills (maintenance tasks) and rewarding effort (reinforcing attempts).

\subsection{Targeting Social Communication Skills during In-Person Sessions}

These six antecedent and consequence PRT strategies can be applied to teach a wide range of social communication skills ranging from first words to engaging in social conversations (Koegel and Koegel 2012). Because many young children with ASD do not initiate frequent functional verbal communication (making requests, asking questions, engaging in reciprocal conversations), a foundational goal of PRT for preschool-aged children with ASD is to enhance social motivation to initiate communication (Koegel et al. 2005). For a child developing early language skills, teaching initiations through making requests is a primary step (Koegel and Koegel 2012).

For example, a PRT therapist could take a toy of interest such as a toy car, check for child interest and attention and then prompt the child to request by saying "car". A child who is not speaking yet may respond with a verbal approximation, "cah". When the child makes a verbal attempt, the clinician provides access to the toy car. If this reinforcer is delivered immediately following the child's verbal response, the child will likely remain motivated to continue engaging and attempting words. As language skills develop, therapists can use the same techniques to teach more advanced skills and address the common problem that children with ASD often do not respond to open-ended questions. For instance, in a play scenario similar to that described above, a therapist could briefly stop the car from rolling. Once the therapist establishes that the child is motivated for the car to continue rolling and is attending, the therapist could ask "what do you want?". When the child makes an appropriate response (e.g., responds "roll car"), the therapist allows the car to continue rolling in the child's direction (contingent reinforcement).

\subsection{Potential Utility of Electronic Devices for PRT Delivery}

Delivery of PRT through electronic devices offers several potential advantages related to establishing effective virtual care including delivery in the child's natural environment, an opportunity to capitalize on intrinsic interest in technology, and potential for clear shared control between child and therapist. These components are outlined in the sections below.

\subsubsection{Delivery in Natural Environment}

One foundational advantage that has been extensively discussed is that the portable nature of modern electronic communication devices allows for families to receive treatment directly in their homes within the child's natural environment. In-home treatment has the benefit of allowing continuous exposure to intervention in natural environments 
(Koegel et al. 1999; Popovic et al. 2020). Delivery in the natural environment (e.g., home) also promotes adaptive and functional communication (Delprato 2001), generalization (Verschuur et al. 1977), and maintenance of skills over time (Moes and Frea 2002; Sheinkopf and Siegel 1998). When treatment is delivered via technology which can be accessed from home, this enables families to access treatment during daily routines and may be beneficial for multiple reasons. For instance, barriers such as time and expense of transportation, cost of parking, and limited childcare for any other children in the family are eliminated. Additionally, delivery of a treatment in a child's natural environment means that skills learned will be directly applicable in the real-life settings where they matter most, rather than having to generalize skills learned in a clinic to the home environment. Telehealth therapy allowed children to respond to opportunities and receive access to reinforcement in their natural home environments, which can increase the likelihood for skills learned during therapy sessions to generalize across environments and people.

\subsubsection{Intrinsic Interest}

Many children may be intrinsically motivated to interact with electronic devices due to a positive learning history associated with tablet or computer use and memories of previous enjoyable experiences using such devices, adding an element of interest that is not typically present during traditional in-person delivery of treatment. For some children, pre-existing interest in technology may have generalized to telehealth sessions that were facilitated on these devices. Since PRT relies on child motivation for successful implementation, any motivation to interact with an electronic device can be useful for attracting initial child attention and can be harnessed to subsequently establish successful child-therapist interactions.

In contrast, another potential advantage for children without much experience using electronic devices could be an unbiased instructional environment, new to children receiving treatment. For some children, the virtual format provided novelty as they learned to interact and play with the therapist in ways not previously experienced and viewed various new electronic activities of interest. Novelty may have increased the likelihood that some children would be motivated to engage in virtual play and eventually treatment.

\subsubsection{Shared Control}

In traditional in-person PRT delivery, shared control between the therapist and child is a crucial component of the motivational contingency. Shared control means that while the teaching always includes an activity of interest to the child (child choice), the adult delivers access to the activity of interest only after the child has made a reasonable attempt to respond (contingent reinforcement). The fact that control is shared in this way is optimal for child motivation because the child's interest is initially captured by the item or activity of interest and with repeated exposure to these types of contingencies, the child learns that responding is required in order to access the item, which motivates greater and greater responsivity to prompts over time.

Treatment delivery via technological devices can be especially effective in facilitating shared control since the therapist can be solely responsible for navigating the virtual platform. As long as the child is interested in some aspect of the virtual interaction (e.g., an action the therapist could perform or an image shared on the screen), the child's access to that activity can be under direct control of the therapist. This can actually result in more consistent control of access to reinforcement compared to a typical in-person interaction, where a child might have difficulty letting go of a toy of interest or grab the item from the therapist's hand. That is, when a similar play interaction is facilitated through a tablet or computer, eliminating the possibility of physical access, many children may demonstrate greater flexibility and patience as they view the therapist manipulating items of interest and online activities.

Treatment via tablets and computers can also provide significant benefit to PRT delivery as therapists maintain shared control during play without negatively impacting 
child motivation. When physically touching the item is not possible, child motivation can shift from attempting to directly access the item to attempting to get the therapist to play with the toy in a specific manner (a more social goal). Therapists are then able to maintain shared control while providing ample language opportunities for the child to express their preferred play scheme. For example, when engaging with an online art activity, the child could be required to make verbal requests ("draw a cat") in order to get the therapist to draw on the screen rather than simply reaching for the marker and drawing themselves as can be done during in-person treatment.

\section{Overview of Objectives and Method}

\subsection{Telehealth Sessions Completed}

Seventeen children ranging from 2 to 5 years old received PRT telehealth sessions over the course of a 13 month period between April 2020 and May 2021. All children had a clinical diagnosis of ASD and had been referred for behavioral treatment to address their associated social communication deficits. These children ranged widely in their language skills from single-word approximations to full sentences, although all children showed at least some communication delay relative to age-based expectations. Of these 17 children, 5 were previously enrolled in a center-based intensive early intervention PRT program and transitioned to virtual visits. The other 12 children started PRT treatment with the telehealth format. Children enrolled in telehealth sessions received 1-5 sessions per week for a duration of 10-55 weeks. Sessions were facilitated by a Registered Behavior Technician (therapist) trained in PRT. A Board-Certified Behavior Analyst (BCBA) provided supervision on a weekly basis, as well as weekly parent training sessions for the first 12 weeks of each family's enrollment. Sessions with RBTs ranged from 45 to $120 \mathrm{~min}$ in duration and were completed via secure video conference software (Zoom). During sessions, the therapists developed a variety of strategies for building child engagement through virtual interactions, allowing the therapists to directly implement virtual PRT with the child over telehealth.

\subsection{Parent Participation in Telehealth Sessions}

Due to the age range of the children who participated in the PRT telehealth program, parents were required to attend and participate in every session. Often, parent direct involvement throughout sessions was necessary initially and then many children became motivated and learned to sustain interaction with the therapists with less parental assistance over time. Because parents were present during clinician-delivered virtual sessions, they actually received more parent training through observation of clinician-delivered PRT sessions than they would have had access to if their child had been attending the inperson classroom therapy environment. This parent involvement led to the unanticipated advantage of more frequent training opportunities for caregivers compared to in-home or center-based models.

\section{Resulting Model: Procedures for Technological Adaptation of PRT}

The section below describes in detail how specific technological components aided in the adaptation of PRT strategies. These technological features made direct implementation possible through the telehealth model, even enhancing the effectiveness of some PRT components. We discuss specific technological tools such as virtual backgrounds, individualized cursor design, camera feeds, and screen sharing, which each have specific utility for delivery of PRT virtually. Table 1 provides additional examples of how these technological adaptations can help therapists execute specific motivational PRT techniques with effects similar to in-person implementation. 
Table 1. Examples of Applying PRT Motivational Strategies via In-Person and Virtual Formats.

\begin{tabular}{|c|c|c|}
\hline $\begin{array}{c}\text { Sample PRT } \\
\text { Motivational Technique }\end{array}$ & $\begin{array}{l}\text { Common Strategies for In-Person } \\
\text { Implementation }\end{array}$ & Virtual Implementation Tools \\
\hline \multirow{4}{*}{ Capturing Child Attention } & - Adult positions self in child's line of sight & $\begin{array}{l}\text { - Use cursor to model play and direct child } \\
\text { attention }\end{array}$ \\
\hline & - Call child name & - Interesting and changing virtual backgrounds \\
\hline & - Hold up item of interest & - Share screen with preferred image or activity \\
\hline & $\begin{array}{l}\text { - Make sounds using the item or play toy's } \\
\text { music }\end{array}$ & $\begin{array}{l}\text { - Annotate circles around specific aspect of image } \\
\text { to direct child attention }\end{array}$ \\
\hline \multirow{3}{*}{ Child Choice } & $\begin{array}{l}\text { - Observe child in play and join whatever } \\
\text { activity interests the child }\end{array}$ & $\begin{array}{l}\text { - Present virtual choice board to communicate } \\
\text { available options }\end{array}$ \\
\hline & $\begin{array}{l}\text { - Hold out two different items for child to } \\
\text { choose }\end{array}$ & - Select a cursor in shape of child's favorite animal \\
\hline & $\begin{array}{l}\text { - Prepare common preferred activities in } \\
\text { advance of session }\end{array}$ & $\begin{array}{l}\text { - Display activity (game, puzzle, book) related } \\
\text { child's special interest through screen share }\end{array}$ \\
\hline \multirow{4}{*}{$\begin{array}{l}\text { Contingent Natural } \\
\text { Reinforcement }\end{array}$} & $\begin{array}{l}\text { - Adult pauses preferred activity and prompts } \\
\text { child to make request }\end{array}$ & - Annotation used to cover image of interest \\
\hline & \multirow{2}{*}{$\begin{array}{l}\text { - Child is motivated to see adult's reaction to a } \\
\text { play act, such as pretend eating }\end{array}$} & $\begin{array}{l}\text { - Camera off, then on, when child is motivated to } \\
\text { see clinician reaction }\end{array}$ \\
\hline & & $\begin{array}{l}\text { - Activate animation or sounds within online } \\
\text { game }\end{array}$ \\
\hline & $\begin{array}{l}\text { - Child needs adult's help to complete a } \\
\text { desired task }\end{array}$ & $\begin{array}{l}\text { - Annotate activities by drawing elements of } \\
\text { interest that are not embedded within activity }\end{array}$ \\
\hline
\end{tabular}

\subsection{Virtual Backgrounds}

The Zoom video call platform used to host telehealth sessions enabled therapists to utilize the virtual background feature during their interactions with patients. The virtual background allows the user to project any image onto the background of their own video. When the feature was utilized, the child could view the therapist simultaneously with their favorite characters or items of interest. Many children with ASD have restricted areas of interest and incorporation of these interests into treatment can be highly effective (Charlop et al. 1990; Charlop-Christy and Haymes 1998). Furthermore, the virtual background feature also enabled therapists to project various locations (grocery stores, airports, underwater scenes), appearing as if they were located in a place of interest throughout the session. Backgrounds could also be instantaneously changed between previously saved images. This feature provided significant therapeutic value unique to the telehealth format, as therapists used virtual backgrounds to direct virtual child interactions and contrive increased social motivation.

In early stages of the telehealth model, virtual backgrounds were a useful tool as the clinical team shaped direct interactions between therapist and the child. For instance, often therapists could capture the child's attention by appearing on screen with their favorite characters. These images helped children orient to the video call during their first sessions. Changing the background helped maintain child interest while providing language opportunities for children to express what image they wanted to see projected next. This also paired therapists with highly preferred items of interest, contributing to successful initial interactions. Establishing a pattern of direct interaction between the therapist and child allowed for the therapist to build rapport and later enabled them to place verbal demands and implement PRT.

Therapists additionally used virtual backgrounds to increase children's social motivation in conversation by incorporating elements of interest that could not otherwise be available as reinforcers. By projecting virtual backgrounds related to preferred locations and routines, many children became increasingly motivated to observe the therapist's 
next location or interaction with the virtual environment, resulting in quality responses to verbal demands. For example, a child interested in trains may be especially motivated to watch their therapist appear to stand in line at the ticket booth, stand on the platform, and choose a seat inside the train using virtual backgrounds. In a typical clinical session, the therapist would not have access to a train station. Even if the therapist and child visited a train station, delivering reinforcement would be limited due to the clinician's limited control of the environment. This highlights the unique advantages of technology and PRT delivery as therapists are able to access reinforcement specifically related to the child's interests while maintaining exceptional levels of control, allowing delivery of the reinforcer at crucial moments.

\subsection{Camera Use}

Cameras provide a live video feed of the child and therapist to a shared platform for all meeting members to view. The therapist's video feed allowed the child to see the therapists play actions, facial expressions, and toys. Over time, some children became interested in directly interacting with their therapist. During these interactions, therapists used exaggerated facial expressions and play acts to reinforce the child's interest. Camera feeds were additionally used to model play with toys and facilitate parallel play. During direct play through the camera, therapists strategically turned their cameras on and off to contrive opportunities for the child to practice asking questions. Examples of this camera use include games of hide and seek or peek-a-boo where the video could be turned off providing an opportunity for the child to ask "Where are you?". The therapist could remain contingent, keeping the camera off, until the child asked independently or responded to the prompt. Immediately after the child asked an appropriate question, the therapist could deliver the natural reinforcer by turning on the camera and reappearing in the child's view.

\subsection{Screen Sharing}

The share screen feature was also embedded into the Zoom video call platform and allowed therapists to share their view of various websites and digital materials with the other attendees in the meeting. Children could watch in real time as the activity was navigated and changed according to their requests, responses, comments, and questions. This tool was vital in facilitating many of the direct play interactions between the therapist and the child. The screen share feature was used to maintain child motivation, utilize online content of interest, and adapt antecedent and consequence PRT strategies for virtual implementation, as detailed below.

\subsubsection{Screen Sharing Benefits: Antecedent Strategies}

A significant benefit of screen sharing is that therapists could utilize an unlimited library of online content related to their patient's interests to enhance treatment. Therapists were able to facilitate age-appropriate games, art projects, books, videos, and integrate images of the patients' favorite characters using this feature. This both improved the longevity of direct child engagement during individual sessions and minimized child boredom related to materials satiation as children were presented with many choices of interest. In an in person setting, clinicians are limited to the toys available in their immediate environment. If a child has a strong preference for a specific subset of toys such as one particular type of dinosaur, the tyrannosaurus, there are likely limited items available that the child will engage with. This repetitive engagement can sometimes limit language opportunities after weeks of treatment and negatively affect a child's motivation as they become satiated with the specific materials. By adapting play to a telehealth format using the screen sharing feature, therapists avoided this source of decreased motivation and enhanced treatment by providing ample child choice. For instance, a therapists could facilitate various tyrannosaurus-themed activities while controlling specific features such as number, size, color, and orientation. This enables a therapist to hold the child's attention by displaying resources related to their restricted interests and strategically editing the 
stimuli throughout play routines. For example, therapists could use a basic online "paint" program to display images of various tyrannosauruses, providing requesting opportunities as the size is edited to make a family. The child can request "small dinosaur" to make a baby and then be contingently reinforced as the therapist shrinks the image. As motivation is sustained and language skills improve, therapists can use these same features to expand the child's use of nouns, verbs, and prepositions with more complex play ideas. For example, the therapist can use the same program to cover the baby dinosaurs with eggs, providing opportunities for increasingly specific requests such as "open the big purple egg" or "crack the spotted egg" while still using the tyrannosaurus to reinforce the request as the character is visible once the egg is contingently removed upon child request or response to prompted request.

\subsubsection{Screen Sharing Benefits: Consequence Strategies}

The screen sharing feature also made direct PRT implementation possible by acting as a vehicle for children to directly experience the tangible outcomes of various consequence strategies. Accurate and instantaneous relaying of virtual events allowed therapists to deliver reinforcement with increased accuracy. In an ideal treatment scenario, reinforcement should be delivered immediately after the target behavior, optimizing the effectiveness of each language opportunity. When delivering tangibles in person, it may not be possible to deliver items within this time frame due to limited availability of the reinforcer or physical complications with locating and delivering the item. Via telehealth, reinforcers could be readily available and consistently delivered within the ideal time frame.

Due to the improved control of reinforcement delivery facilitated by the screen share feature, verbal behavior could also be shaped more effectively through virtual implementation of differential reinforcement. Children receiving differential reinforcement receive a less valuable reinforcer, such as a single ball, following an easier verbal (maintenance) task. For engaging in difficult verbal (acquisition) tasks, the child receives a more valuable reinforcer, such as three balls at once. Through telehealth PRT implementation, the difference between maintenance and acquisition tasks could be emphasized by immediate and obvious differences in contingent reinforcement.

Many interactive online games are available for young children (often hosted by popular children's television program websites). When therapists were able to use screen sharing to facilitate a child's viewing of an online game (rather than having the child operate the game directly themself), contingent and differential reinforcement could be naturally provided by the therapist. Ideal games were open ended scenes incorporating familiar characters and interacting animated elements that could be controlled by clicking specific features. For example, while participating in an online game centered around a pretend bath time, the therapist controlling the actions of the electronic game could make a single bubble appear for a child who likes bubbles, immediately following a child's response to the prompted request (maintenance task), "bubble". The child watches the bubble appear on screen, watches it float through the scene, and hears the corresponding sound effect. When the child independently initiates the request "bubble" (acquisition task), therapist can continuously deliver many bubbles allowing the child to watch them appear and hear the continued sound effects. This consequence is enjoyable for the child and can be easily used to shape independent requesting as an obviously preferred outcome occurs immediately after the child's appropriate response. This activity could be adapted to teach more complex language, such as attending to multiple features of an object. For instance, the therapist could pause the activity and prompt the child to make a feature-specific request ("big green bubble") and manipulate the virtual stimulus accordingly contingent on the child's response. While execution of this type of routine via telehealth requires access to an online game which allows the therapist to make images appear directly on the child's screen, many such games currently exist for young children and are freely available. The online format also allows the therapist to deliver the bubble reward immediately and consistently, which can be difficult to do in-person. 


\subsection{Custom Cursors}

Another useful tool for directing child attention while screen sharing online activities was the cursor. Cursors could be moved repeatedly around a specific element on the screen in effort to draw the child's attention. Beyond using motion to direct child attention, therapists used internet browser extensions to customize their cursors. These extensions provided options to change the size, color, and design of the cursor. Therapists were able to make their cursors visually obvious while integrating almost any child interest. Integrating this technological tool increased therapists' accuracy when implementing antecedent strategies, ensuring the child was attending (child attention) to the appropriate stimuli related to the corresponding verbal task (clear opportunities).

During in-person treatment, physical proximity is commonly used to assess child attention and create clear opportunities. For example, when playing with a marble track, the therapist may use an exaggerated movement to lower the marble to the track and pause right before dropping it in. If the child is looking at the marble (child attention) the pause creates a clear opportunity for the child to request "put the marble in" to continue the play routine. While delivering treatment virtually, therapists instead rely on their cursor to direct the child's attention and model play routines. For instance, during virtual play, a therapist may model play within a music game by moving the cursor across each bar of a virtual xylophone and clicking to hear the note. If the child appears interested in the activity, the therapist could pause the play routine by hovering the cursor over the next note. This creates a clear opportunity, similar to in-person play with the marble track. The child could initiate the request "play the xylophone" in order to continue the activity. As long as child interest can be secured with a preferred image, customized cursors make visual attending and tracking across different backgrounds easier for young children. Integrating designs of strong individual interest to the child can further increase a child's enjoyment while watching the cursor. For instance, selecting a cursor in the shape of a child's favorite animal could secure greater child attention to the screen.

\subsection{Annotation Feature}

Annotation is another useful feature available through the Zoom video call platform which enabled therapists to mark or draw directly onto any webpage image or application being shared with the other meeting members through screen share. This feature was versatile and helped therapists direct the child's attention in addition to setting up contingencies and delivering natural reinforcement.

Therapists often used the annotation feature to direct attention to a specific area of the screen by drawing circles or arrows pointing to the object of interest. Utilized within antecedent strategies, a therapist could use the annotation feature to direct child attention and create clear opportunities during an online reading activity. A therapist could pause the story and circle a character while asking "what is she doing?" In this instance the therapist used the annotation feature to help the child identify which character to talk about. Additionally, a therapist could present the same question without circling the character. If the child does not respond, the therapist could then circle the character while representing the question, making the verbal task easier and increasing the likelihood of child responding. As long as the therapist remains contingent in delivering the natural reinforcer, continuing with the story, this does not compromise the quality of the opportunity.

The annotation feature was also an effective tool in contriving successful child opportunities to ask questions. Therapists used the annotation feature to draw opaque boxes over images of interest, gaining control of the image's visibility. The therapist could prompt the child to ask "what's that?". When the child responds, the therapist would remove the box delivering contingent reinforcement by allowing the child to see the desired image. In this scenario, the annotation feature was used throughout the whole opportunity as the therapist established shared control and contingently allowed access to the natural reinforcer.

Finally, the annotation feature was used to deliver the PRT consequence strategy, natural reinforcement. While participating in online games such as dress up, children 
commonly displayed significant motivation when engaging with activities related to their interests. During these activities, children often initiated requests outside of the game's available options. If a child requested "purple glasses", the therapist could use the annotation feature to draw purple sun glasses on the character. Annotation allowed therapists to reinforce a greater variety of independent initiated requests and further individualize activities to each child's interest.

\subsection{Virtual Choice Boards}

Virtual choice boards were an additional technique that allowed therapists to further integrate child choice within PRT telehealth sessions despite the adult-led nature of some online activities. Choice boards are a type of visual aide often used during in-person treatment to provide a visual representation of available activities, songs, or toys. These options can be represented by words, individual icons, or photos arranged in a grid on a single piece of paper or board. Choice boards allow children to view all options of interest at once, even if the items themselves are not visible within the immediate environment. Therapists use choice boards to assess child preference and choose effective reinforcers. Choice boards were adapted to the virtual platform to help therapists assess a child's preference despite the therapist's inability to observe a child navigate the virtual environment as they would in an in-person setting. Therapists maintained documents with images of preferred and new online activities related to each child's interests. These tools were displayed periodically throughout session and proved helpful in mitigating diminishing child motivation by adapting PRT antecedent strategies.

Virtual choice boards were utilized strategically throughout telehealth sessions to integrate child choice during critical moments of fragile child engagement. During a standard $60 \mathrm{~min}$ telehealth session, children commonly transitioned between a few activities specific to their interests. After a prolonged period of engagement, many children become satiated with an activity, often manifesting behaviorally as fidgeting in their chair, looking away from the screen, and decreased responding. According to antecedent PRT principles, if a child is no longer motivated to participate in any given activity, the clinician's focus shifts to reestablishing motivation for another activity before placing verbal demands. In-person, a therapist can assess motivation without placing verbal demands by physically offering a toy or modeling novel play. Signs of child motivation for the new items include coming over to the area, looking, and reaching for items. When implementing PRT via telehealth, virtual choice boards were used in leu of other proximity play techniques described above. Keeping in mind the child's wavering motivation to continue the virtual interaction, therapists displayed choice boards while minimizing verbal demands and waiting for signs of interest in any of the options. By accepting any sign of interest, prolonged looking or pointing, the therapist can open the activity to further establish child interest before placing verbal demands. In instances where a child does not choose an activity, the therapist can attempt to entice child interest by starting one potential activity and modeling play free of verbal demands, similar to in-person practices.

\section{Implications and Future Directions}

Delivery of PRT via telehealth has implications for clinicians as well as their relationships with the families and children they work with. This section outlines additional benefits related to training opportunities for both therapists and parents.

\subsection{Parent-Therapist Interactions}

The telehealth model required parents and therapists to collaborate in order to create clear opportunities for children to communicate and receive immediate reinforcement, as therapists did not have direct access to the children. Thus, high levels of parent-therapist rapport, communication and collaboration were pertinent in optimizing treatment delivery. Parents and therapists often worked together during virtual sessions in assessing child motivation, proving clear prompts, and delivering reinforcement. This was especially 
true for children with limited verbal ability and play skills and those who did not directly attend to the therapist or screen at the onset of treatment. Therapists often needed to guide parents in gaining shared control through manipulating toys or presenting activities. Throughout both play with tangibles and virtual play through the video call, parents also relayed important information about child interest and motivation to the therapist. Parents described child attention cues including the child's body language and eye gaze when the therapist's visibility of the child and environment were impaired by the limited view of the camera. This level of collaboration allowed parents and therapists to build stronger relationships while allowing children and families to access ecologically valid treatment, considering the setting in which treatment occurred and the parents' active role in delivering treatment.

Future research will be necessary to further investigate these potential benefits; however, greater opportunities to work with parents might potentially result in faster skill building for therapists, specifically in the areas of rapport building, navigating difficult conversations, coaching parents through episodes of challenging behavior, and providing feedback about parent implementation of PRT strategies. Therapists in our program provided anecdotal reports related to improved job satisfaction as they had more frequent exposure to family members and regularly received parental feedback regarding their children's progress at home. It is possible that these factors could help therapists to remain invested in their work and feel that they are making a positive impact. This is consistent with findings of a greater body of research which suggests that positive interactions with parents and parent-reported child progress contribute to job satisfaction in the field of behavioral treatment (Novack and Dixon 2019). It will be important for future research to investigate whether level of parent involvement in direct treatment delivery helps parents meet fidelity faster, increases job satisfaction, and has an impact on therapist intent to pursue further training in ABA. Further, it is important for future telehealth models to consider the implications for RBT training if parent-therapist interactions become a routine or required part of most telehealth sessions.

\subsection{Supervisor-Therapist Interactions}

Traditional in-person ABA treatment requires ongoing supervision and treatment modification from a Board-Certified Behavior Analyst (BCBA) supervisor. During scheduled supervision sessions, a BCBA observes the therapist working directly with the child and provides coaching and feedback. In the telehealth model, the supervisor joined virtual sessions and observed live as therapists implemented PRT with the child or collaborated with parents to implement PRT. In many instances, feedback and suggestions were delivered through Zoom's private chat function, which proved instrumental in helping therapists develop skills related to interacting, coaching, and communicating with both parents and children online. This tool allowed the supervisor to give immediate feedback and solutions as therapists facilitated play activities, parent interactions, and behavior management strategies. The private, written delivery of feedback allowed for minimal treatment disruption and made it more feasible for supervisors to provide therapists with discrete feedback related to parent coaching. Finally, private messaging presented major benefits as therapists received clinical guidance without losing an opportunity to practice communicating directly with the child or parent.

Access to immediate supervisory feedback and reinforcement was a key component in the adaptation of PRT principles discussed above, which may have had significant implications for clinician experiences. Recent literature evaluating the experiences of behavior analysts and therapists during the COVID-19 pandemic suggests that perceived supervisor support is correlated with job satisfaction and burnout rates (Jimenez-Gomez et al. 2021; Novack and Dixon 2019). Respondents also reported that the use of technology has been paramount to sharing relevant information and receiving ongoing support, ultimately promoting feelings of workplace security and productivity. Future research should explore 
how to optimize the use of this virtual supervision format both during online treatment and potentially as a supplement to in-person supervision.

Another unanticipated benefit of our abrupt transition to telehealth treatment delivery was that the need to develop a new treatment model facilitated intense collaboration between supervisors and therapists, which was rewarding for both. Supervisors and therapists took a team approach to innovating and adapting PRT procedures to be delivered in the novel virtual context. This provided collaboration opportunities that are not usually available in traditional contexts, as much is already "known" about effective treatment for young children with ASD. Finding ways to foster innovation and novel collaborations during ongoing treatment delivery could be beneficial for professional engagement and fulfillment of both therapists and supervisors and further research in this area could be beneficial to both clinicians and the families they serve.

\section{Conclusions}

This paper described how therapists implementing Pivotal Response Treatment with young children with ASD modified the procedures for telehealth delivery and how they were able to capitalize on key aspects of the video conference technology to enhance a treatment which has previously only been delivered in-person. Through the use of digital resources, therapists were able to engage children through presentation of their preferred interests in varied formats and with immediacy, which allowed for therapists to capitalize on the digital versions of PRT techniques like following a child's lead, establishing shared control, and providing contingent natural reinforcement. In the in-person treatment settings, resources related to child-specific interests can be more limited.

Given our initial observed positive response to virtual PRT delivery from many participating children with ASD and their parents, future research to refine best practices and quantify the effects of the treatment on child communication is certainly warranted. Our hope is that this conceptual article will spark interest in empirical investigation of virtual PRT implementation procedures. A critical next step will be to empirically evaluate these procedures in a controlled design. A particularly important direction for future research will be to explore which target skills can be taught best in a virtual format and how child and family characteristics may be used to determine the optimal mix of in-person and video-based learning. If effectiveness of virtual PRT delivery could be established, the ability to deliver some ASD treatment via telehealth could allow many more children access to evidence-based and necessary care.

Although it is surprising that children with significant social communication deficits could learn to communicate meaningfully through virtual interaction with a therapist, the use of technology in treatment yielded specific advantages for motivating children which are not typically available during in-person sessions. First, for many participating children, the novelty and reinforcing properties of screen-based interactions were inherently motivating. Many children showed interest in electronics, which allowed for less time needed to build rapport and pair therapy with reinforcement prior to presenting learning trials. Furthermore, therapists were able to quickly incorporate an almost unlimited library of instructional materials to secure child interest and provide natural reinforcement for appropriate social communication skills. Finally, the physical separation between child and therapist allowed for highly effective shared control, a critical aspect of the motivational contingencies used in PRT. Specifically, the therapist could use technology tools like screen sharing to optimize the combination of child control and adult control, without the child being distracted by trying to get physical control of the item themself.

Some key challenges observed included when children were minimally interested in the therapists' actions on screen and for children with very low rates of verbal speech and extremely short attention span. While therapists were typically able to eventually direct a child's attention to themself or an activity on the screen where the therapist had control of the action, there were some instances where a child was initially more interested in touching or manipulating the electronic device themself. Anecdotal observation suggests 
that children with specific strong interests in characters or specific screen-based activities may do better with this type of online treatment delivery. Future research will be needed to investigate this hypothesis further. In addition, it is likely that intensity similar to that which is typical for in-person sessions (e.g., 10-25 h per week of direct therapy) may be too much to expect of young children in the online format. Investigation into the optimal number of hours or optimal combination of in-person and virtual treatment is strongly needed. Finally, future implementation of this model will also depend on family access to technological devices and internet bandwidth sufficient for video conference sessions.

The lessons learned from providing direct behavioral therapy in a virtual format also have widespread implications for distance learning and other situations where adults need to engage children virtually. For instance, deliberately incorporating child-specific interests into virtual background design, cursor selection, and screen sharing content can effectively capture a child's attention. Establishing clear behavioral contingencies whereby the adult can deliver a positive consequence immediately following a child's attempt to respond, provides opportunity for clear feedback to support efficient learning. Given that so many aspects of electronic device use are inherently interesting to young children, the systematic use of this technology to prompt and naturally reinforce appropriate social engagement and responding is highly promising. In addition, future implementation of virtual learning modules should consider strategies for successful collaboration between parents and therapists to facilitate optimal outcomes for parent implemented treatment in home and community settings.

During the COVID-19 pandemic, major concerns have been raised about the effectiveness of online learning for young children, even those who are typically developing (Dong et al. 2020). Many teachers and early childhood educators struggled to engage young children online to practice meaningful and developmentally appropriate social and pre-academic skills. Even as in-person instruction again becomes the norm, the opportunity to engage and educate children at a distance will nevertheless remain an important tool for increasing access to educational opportunity regardless of geographic location, transportation, and other potential barriers. Research incorporating motivational behavioral principles drawn from PRT could make an important contribution to how educators and therapists of all types effectively engage children online and motivate meaningful learning.

Author Contributions: Project administration and writing-original draft preparation, D.M.W. and C.A.-P.; conceptualization, supervision, and writing-review and editing, D.M.W. and G.W.G. All authors have read and agreed to the published version of the manuscript.

Funding: This work was supported in part by The Teresa and Charles Michael Fund for Autism Research and Education at Stanford.

Institutional Review Board Statement: Not applicable. This concept paper describes intervention implementation in a clinical program.

Informed Consent Statement: Not applicable. This concept paper does not report research findings or outcomes from human subjects.

Acknowledgments: We gratefully acknowledge the participating families and the therapists who contributed to this project, including Maria Bundang and Judyth Figueroa. We would also like to thank Antonio Hardan and members of the Stanford Autism and Developmental Disorders Research Program (ADDRP) for their support.

Conflicts of Interest: The authors declare no conflict of interest.

\section{References}

Bruinsma, Yvonne, Mendy Minjarez, Laura Schreibman, and Aubyn Stahmer. 2019. Naturalistic Developmental Behavioral Interventions for Autism Spectrum Disorder. Towson: Brookes Publishing.

Charlop, Marjorie H., Patricia F. Kurtz, and Fran Greenberg Casey. 1990. Using aberrant behaviors as reinforcers for autistic children. Journal of Applied Behavior Analysis 23: 163-81. [CrossRef]

Charlop-Christy, Marjorie, and Linda Haymes. 1998. Using objects of obsession as token reinforcers for children with autism. Journal of Autism and Developmental Disorders 28: 189-98. [CrossRef] [PubMed] 
Cihon, Joseph H., Julia L. Ferguson, Matthew Lee, Justin B. Leaf, Ron Leaf, and John McEachin. 2021. Evaluating the cool versus not cool procedure via telehealth. Behavior Analysis in Practice, 1-9. [CrossRef]

Delprato, Dennis J. 2001. Comparisons of discrete-trial and normalized behavioral language intervention for young children with autism. Journal of Autism Developmental Disorders 31: 315-25. [CrossRef]

Dong, Chuanmei, Simin Cao, and Hui Li. 2020. Young children's online learning during COVID-19 pandemic: Chinese parents' beliefs and attitudes. Children and Youth Services Review 118: 105440. [CrossRef]

Gengoux, Grace W., Daniel A. Abrams, Rachel Schuck, Maria Estefania Millan, Robin Libove, Christina M. Ardel, Jennifer M. Phillips, Melanie Fox, Thomas W. Frazier, and Antonio Y. Hardan. 2019. A pivotal response treatment package for children with autism spectrum disorder: An RCT. Pediatrics 144: e20190178. [CrossRef]

Hardan, Antonio Y., Grace W. Gengoux, Kari L. Berquist, Robin A. Libove, Christina M. Ardel, Jennifer Phillips, Thomas W. Frazier, and Mendy B. Minjarez. 2015. A randomized controlled trial of Pivotal Response Treatment Group for parents of children with autism. Journal of Child Psychology and Psychiatry 56: 884-892. [CrossRef]

Jimenez-Gomez, Corina, Gargi Sawhney, and Kristin M. Albert. 2021. Impact of COVID-19 on the Applied Behavior Analysis Workforce: Comparison across Remote and Nonremote Workers. Behavior Analysis in Practice, 1-10. [CrossRef]

Koegel, Robert L., and Lynn K. Koegel. 2012. The PRT Pocket Guide: Pivotal Response Treatment for Autism Spectrum Disorders. Baltimore: Brookes Publishing Company.

Koegel, Robert L., and Lynn K. Koegel, eds. 2019. Pivotal Response Treatment for Autism Spectrum Disorders, 2nd ed. Baltimore: Paul H. Brookes.

Koegel, Lynn Kern, Robert L. Koegel, Kristen Ashbaugh, and Jessica Bradshaw. 2014. The importance of early identification and intervention for children with or at risk for autism spectrum disorders. International Journal of Speech-Language Pathology 16: 50-56. [CrossRef]

Koegel, Lynn K., Robert L. Koegel, and Lauren I. Brookman. 2005. Child-initiated interactions that are pivotal in intervention for children with autism. In Psychosocial Treatments for Child and Adolescent Disorders: Empirically Based Strategies for Clinical Practice. Edited by E. D. Hibbs and P. S. Jensen. Washington: American Psychological Association, pp. 633-57.

Koegel, Lynn, Robert Koegel, Yifat Shoshan, and Erin McNerney. 1999. Pivotal Response Intervention II: Preliminary long-term outcome data. Research and Practice Persons with Severe Disabilities 24: 186-98. [CrossRef]

Lindgren, S., D. Wacker, A. Suess, K. Schieltz, K. Pelzel, T. Kopelman, J. Lee, P. Romani, and D. Waldron. 2016. Telehealth and autism: Treating challenging behavior at lower cost. Pediatrics 137: S167-75. [CrossRef] [PubMed]

McGarry, Elizabeth, Ty Vernon, and Anisha Baktha. 2020. Brief report: A pilot online pivotal response treatment training program for parents of toddlers with autism spectrum disorder. Journal of Autism and Developmental Disorders 50: 3424-3431. [CrossRef]

Moes, Doug R., and William D. Frea. 2002. Contextualized behavioral support in early intervention for children with autism and their families. Journal of Autism Developmental Disorders 32: 519-33. [CrossRef] [PubMed]

Mohammadzaheri, Fereshteh, Lynn Kern Koegel, Mohammad Rezaee, and Seyed Majid Rafiee. 2014. A randomized clinical trial comparison between pivotal response treatment (PRT) and structured applied behavior analysis (ABA) intervention for children with autism. Journal of Autism and Developmental Disorders 44: 2769-2777. [CrossRef] [PubMed]

Mouga, Susana, Bárbara Regadas Correia, Cátia Café, Frederico Duque, and Guiomar Oliveira. 2020. Language Predictors in Autism Spectrum Disorder: Insights from Neurodevelopmental Profile in a Longitudinal Perspective. Journal of Abnormal Child Psychology 48: 149-61. [CrossRef] [PubMed]

National Autism Center. 2015. Findings and Conclusions: National Standards Project, Phase 2. Available online: www. nationalautismcenter.org (accessed on 19 January 2019).

National Research Council. 2001. Educating Children with Autism. Washington: The National Academies Press. [CrossRef]

Nefdt, Nicolette, Robert Koegel, George Singer, and Michael Gerber. 2010. The Use of a Self-Directed Learning Program to Provide Introductory Training in Pivotal Response Treatment to Parents of Children with Autism. Journal of Positive Behavior Interventions 12: 23-32. [CrossRef]

Novack, Marlena N., and Dennis R. Dixon. 2019. Predictors of burnout, job satisfaction, and turnover in behavior technicians working with individuals with autism spectrum disorder. Review Journal of Autism and Developmental Disorders 6: 413-21. [CrossRef]

Pacia, Cressida, Jennifer Holloway, Ciara Gunning, and Helena Lee. 2021. A Systematic Review of Family-Mediated Social Communication Interventions for Young Children with Autism. Review Journal of Autism and Developmental Disorders, 1-27. [CrossRef]

Popovic, Svetlana C., Elizabeth M. Starr, and Lynn K. Koegel. 2020. Teaching Initiated Question Asking to Children with Autism Spectrum Disorder Through a Short-Term Parent-Mediated Program. Journal of Autism and Developmental Disorders 50: 3728-38. [CrossRef]

Posar, Annio, and Paola Visconti. 2019. Long-term outcome of autism spectrum disorder. Turkish Archives of Pediatrics/Türk Pediatri Arşivi 54: 207-12. [CrossRef] [PubMed]

Schreibman, Laura, and Aubyn C. Stahmer. 2014. A randomized trial comparison of the effects of verbal and pictorial naturalistic communication strategies on spoken language for young children with autism. Journal of Autism and Developmental Disorders 44: 1244-51. [CrossRef] [PubMed]

Schreibman, Laura, Geraldine Dawson, Aubyn C. Stahmer, Rebecca Landa, Sally J. Rogers, Gail G. McGee, and Connie Kasari. 2015. Naturalistic developmental behavioral interventions: Empirically validated treatments for autism spectrum disorder. Journal of Autism and Developmental Disorders 45: 2411-28. [CrossRef] [PubMed] 
Sheinkopf, Stephen J., and Bryna Siegel. 1998. Home-based behavioral treatment of young children with autism. Journal of Autism and Developmental Disorders 28: 15-23. [CrossRef] [PubMed]

Simacek, Jessica, Marianne Elmquist, Adele F. Dimian, and Joe Reichle. 2021. Current trends in telehealth applications to deliver social communication interventions for young children with or at risk for autism spectrum disorder. Current Developmental Disorders Reports 8: 15-23. [CrossRef]

Steinbrenner, Jessica R., Kara Hume, Samuel L. Odom, Kristi L. Morin, Sallie W. Nowell, Brianne Tomaszewski, Susan Szendrey, Nancy S. McIntyre, Şerife Yücesoy-Özkan, and Melissa N. Savage. 2020. Evidence-Based Practices for Children, Youth, and Young Adults with Autism. Chapel Hill: The University of North Carolina at Chapel Hill, Frank Porter Graham Child Development Institute, National Clearinghouse on Autism Evidence and Practice Review Team.

Verschuur, Rianne, Robert Didden, Russell Lang, Jeff Sigafoos, and Bibi Huskens. 1977. An implicit technology of generalization. Journal of Applied Behavior Analysis 10: 349-67.

Verschuur, R., R. Didden, R. Lang, J. Sigafoos, and B. Huskens. 2014. Pivotal Response Treatment for Children with Autism Spectrum Disorders: A Systematic Review. Review Journal of Autism and Developmental Disorders 1: 34-61. [CrossRef]

Vismara, Laurie A., Carolyn E. B. McCormick, Amy L. Wagner, Katernia Monlux, Anna Nadhan, and Gregory S. Young. 2016. Telehealth parent training in the Early Start Denver Model: Results from a randomized controlled study. Focus on Autism and Other Developmental Disabilities 33: 67-79. [CrossRef] 